\title{
A INFLUÊNCIA DA VINCULAÇÃO NOS SENTIMENTOS DE SOLIDÃO NOS JOVENS UNIVERSITÁRIOS: IMPLICAÇÕES PARA A INTERVENÇÃO PSICOLÓGICA
}

\author{
Marta Teixeira Bastos ${ }^{1}$ \\ Maria Emília Costa ${ }^{2}$
}

\begin{abstract}
Resumo: Baseado numa perspectiva desenvolvimental da vinculação, o presente estudo tem como objectivo fundamental estudar a influência da vinculação na vivência do sentimento de solidão. Assumindo que a vinculação pode operar como um factor de risco (vinculação insegura) ou como um factor protector (vinculação segura) do desenvolvimento, pretende-se compreender como é que a segurança da vinculação interfere na vivência do sentimento de solidão, em dois dos principais contextos relacionais dos jovens: a relação com os pais e a relação amorosa. Os resultados demonstraram que uma maior segurança na vinculação está associada a níveis menores de solidão, em ambos os contextos relacionais. A compreensão da interacção existente entre a vinculação formada e os sentimentos de solidão abre caminho a novas hipóteses de intervenção na experiência de solidão.
\end{abstract}

Palavras-chave: vinculação, solidão, intervenção psicológica, jovens adultos

The influence of attachment in young adults' loneliness feelings: implications for psychological intervention (Abstract): Attachment can work as a risk factor (attachment security) or as a protective factor (attachment insecurity) toward adolescent development. Based on a life-span attachment perspective, the following study attempts to explore the influence of attachment security in loneliness felt in parental and romantic relationships. As expected, higher security of attachment was related to lower levels of loneliness in both interpersonal contexts. Implications of these findings in designing therapeutic interventions according to the individual needs of the lonely are discussed.

\footnotetext{
${ }^{1}$ Aluna de Doutoramento da Faculdade de Psicologia e Ciências da Educação da Universidade do Porto.

${ }^{2}$ Professora Associada com Agregação da Faculdade de Psicologia e Ciências da Educação da Universidade do Porto.
}

PSICOLOGIA, Vol. XVIII (2), 2005, Edições Colibri, Lisboa, pp. 33-56 


\section{Introdução}

Embora o interesse pelo fenómeno da solidão seja muito antigo, até referido no livro do Genesis, o ênfase pelo seu estudo científico despoleta na segunda metade do séc. XX, sob o interesse de investigadores como Sullivan (1953), From-Reichman (1959), Moustakas (1961), Weiss (1973) e Peplau e Perlman (1982), assumindo um marco fundamental a criação da primeira escala de avaliação da solidão - University of California Loneliness Scale, UCLA (Russell, Peplau \& Ferguson, 1978).

Um padrão estável de solidão constitui uma ameaça forte ao funcionamento psicológico e à saúde mental do indivíduo (McWhirter, 1990). A solidão aparece associada a uma variedade de problemas, como timidez, depressão, vinculações inseguras, ansiedade social e autoconsciência, doenças físicas, suicídio, consumo de álcool, agressão e insucesso académico, entre outros (Perlman \& Landolt, 1999). A solidão aparece ainda associada a sensações crónicas de cansaço, dor e tensão (Gerstein \& Tesser, 1987).

Segundo Bérubé e Joshi (1998), os jovens constituem um dos grupos mais susceptíveis de vivenciar a solidão. Segundo Costa (1991), "Quando o jovem não é capaz de ter relações íntimas com outros (...) as suas relações tenderão a ser estereotipadas com um profundo sentimento de isolamento" (pp. 38). Brage, Meredith, e Wooward (1993), Jong-Gierveld (1987) e Peplau e Perlman (1982) observaram que a adolescência é o período desenvolvimental onde há um risco elevado para o aparecimento de sentimentos fortes de solidão, sendo nesta idade que a solidão aparece com maior frequência.

Uma vez que esta investigação se situa no âmbito das relações que o jovem adulto constrói com os seus pais e o par amoroso, a teoria da vinculação, que procura compreender a formação e o desenvolvimento da personalidade no contexto das relações afectivas e significativas, parece ser a mais pertinente para explicar a possível influência que estas experiências relacionais terão na forma como o jovem percepciona o fenómeno da solidão, nomeadamente qual o grau em que experiencia solidão em cada uma destas relações, e qual a atitude que assume face à solitude.

É objectivo último deste trabalho produzir algumas pistas para a intervenção psicológica na solidão, já que esta problemática é cada vez mais frequente nos jovens portugueses (ver estudo da OMS [1997/98] sobre "Comportamentos Saudáveis de Crianças e Jovens em Idade Escolar"). 


\begin{abstract}
A Solidão
A solidão tem sido definida como "uma experiência desagradável que ocorre quando a rede de relações sociais do indivíduo apresenta um défice significativo, seja qualitativo seja quantitativo" (Perlman \& Peplau, 1981, pp. 31). Segundo esta perspectiva, a solidão é conceptualizada como um construto unidimensional, resultante da discrepância entre as relações sociais desejadas pelo indivíduo e as actuais (Peplau \& Perlman, 1982; Russel, Peplau \& Cutrona, 1980). Segundo esta teoria, a solidão pode resultar de mudanças na rede social actual do indivíduo ou de mudanças nos seus desejos ou expectativas relativamente às relações sociais. A UCLA Loneliness Scale (Russell, Peplau \& Cutrona, 1980), para adultos, e o Illinois Loneliness Questionnaire (ILQ, Asher, Hymel, \& Renshaw, 1984), para crianças, são exemplos de instrumentos desenvolvidos nesta linha de pensamento, que apenas analisam a intensidade da experiência de solidão, desprezando o estudo das suas causas ou origens, da sua duração (temporária ou crónica, solidão traço ou estado) e de possíveis modos de a reduzir.

Contrária a esta corrente, a Teoria das Necessidades Sociais (Bowlby, 1973; Sullivan, 1953; Weiss, 1973) conceptualiza a solidão como uma resposta a um défice relacional, percepcionada quando as necessidades sociais dos indivíduos não são satisfeitas no seio dos seus relacionamentos. Segundo esta teoria, se uma relação não é capaz de satisfazer a totalidade das necessidades sociais (vinculação, integração social, orientação, reconhecimento pessoal, aliança segura e nurturance), o indivíduo irá experienciar solidão. Esta conceptualização sugere que a solidão é um fenómeno multifacetado, que pode apresentar diversas manifestações (Belcher, 1973; Schmidt, 1976).

Enquadrado nesta corrente, Weiss (1973) define dois tipos de solidão: a solidão social e a solidão emocional. A solidão social implica a percepção de um défice na rede de relações sociais do indivíduo. Ao originar sentimentos de exclusão, marginalidade e aborrecimento, a solidão move o indivíduo a procurar aumentar ou desenvolver a sua rede social de apoio. A solidão emocional surge quando o indivíduo percepciona a ausência de uma relação íntima, emocional ou vinculativa, onde se possa sentir aceite, seguro, compreendido e protegido. Este sentimento move-o a procurar o estabelecimento de uma relação de vinculação satisfatória, ou ao restabelecimento de uma que tenha perdido. Embora conceptualmente diferentes, estes dois tipos de solidão podem ocorrer simultaneamente, não sendo possível, porém, diminuir os défices percepcionados numa forma de solidão, substituindo-os por outros tipos de relações. Exemplificando, o facto de um indivíduo possuir uma rede de apoio social satisfatória não o impede de sentir solidão emocional, causada pela ausência de uma relação de intimidade.
\end{abstract}


A presente investigação procura estudar a solidão nos jovens, adoptando esta perspectiva multidimensional da solidão. Assumindo como base o trabalho de Marcoen, Goossens e Caes (1987), procura-se avaliar os diferentes níveis de solidão que o jovem percepciona em dois dos seus principais contextos interpessoais de vida (relação com os pais e relação com o par amoroso), e analisar o tipo de atitude que ele assume relativamente ao estado de solitude. A solitude, definida por Marcoen e Goossens (1993) como "o uso activo e construtivo do tempo passado só" (pp. 198), pode ser conceptualizada como uma necessidade desenvolvimental paralela à necessidade de se estabelecer relações interpessoais (Buchholz \& Catton, 1999). Winnicott (1957) define solitude como uma capacidade desenvolvida na infância para se estar sozinho, fortemente influenciada pela qualidade das relações com as figuras de vinculação e fundamental a um processo maturacional saudável.

A solidão na relação parental resulta da percepção de um défice no relacionamento com os pais, que não são capazes de satisfazer as necessidades de proximidade emocional, afecto ou segurança, dos seus filhos. Estes sentimentos de solidão podem resultar de dificuldades no relacionamento entre pais e filhos, fruto de características pessoais ou de alterações ambientais (por exemplo, mudança de residência, entrada na faculdade, tempo dedicado ao trabalho) (Goossens e Marcoen, 1999).

A solidão romântica (definida por DiTommaso e Spinner, 1997) surge associada à incapacidade em estabelecer uma relação amorosa satisfatória, quer porque o indivíduo ainda não conseguiu estabelecer nenhuma relação, quer porque a relação de namoro existente não assume o nível de intimidade e proximidade emocional desejado. Mais uma vez, esta solidão pode resultar de diferenças individuais ou, por exemplo, do facto de os elementos do par viverem espacialmente distantes um do outro.

Por último, a avaliação da percepção que os jovens têm do tempo que passam sós procura compreender qual a atitude assumida face à experiência de solitude (procura versus aversão). A "procura de solitude" implica a experiência de sentimentos positivos quando se está só e está positivamente correlacionada com a construção de uma identidade autónoma e a manutenção do equilíbrio psicológico (Larson, 1990). A "aversão à solitude" avalia o medo de estar só ou a sensação de mal-estar na ausência da companhia de outras pessoas. Estas duas atitudes não devem ser conceptualizadas como pólos opostos de um mesmo continuum, mas sim como representando construtos distintos para o indivíduo (Goossens, Marcoen, Hees \& Woestijne, 1998) 


\section{A Vinculação}

A Teoria da Vinculação afirma a necessidade humana universal de os indivíduos construírem relações afectivas de proximidade. A investigação da vinculação na idade adulta proporcionou a descentração da análise de variáveis externas e observáveis ("situação estranha") para aspectos internos e representacionais da vinculação. A teoria de Bowlby tem sido aplicada ao estudo das relações entre pais e filhos (Greenberg, Siegal \& Leitch, 1984), das relações românticas (Collins \& Read, 1980; Hazan \& Shaver, 1987) e à definição de diferentes protótipos de vinculação no jovem e adulto (Bartholomew \& Horowitz, 1991; Main, Kaplan \& Cassidy, 1985).

Segundo Bowlby (1973), os modelos internos dinâmicos são utilizados pelo sujeito para prever a disponibilidade e responsividade do outro para responder às suas necessidades de apoio e protecção, e orientá-lo na construção das suas relações de proximidade. Segundo Bowlby (1988), à medida que a criança e o jovem se movem pelas diferentes trajectórias desenvolvimentais, as experiências vividas nesses diferentes contextos provocam oscilações na segurança da vinculação, podendo levar a que existam diferenças nos modelos internos dinâmicos das relações vividas em cada domínio: por exemplo, a pessoa pode actuar e percepcionar a relação parental de determinado modo, mas de maneira diferente a relação com os pares.

$\mathrm{Na}$ adolescência, assistimos a um período de revisão dos modelos de vinculação (modelo de representação do self e do outro), dado o seu confronto com outras experiências de vinculação estabelecidas ao longo do ciclo de vida, dentro e fora do contexto familiar, e pela emergência das capacidades cognitivas no jovem e adulto. Se, na infância, a família se constitui como o contexto mais significativo de vinculação, à medida que o adolescente se vai desenvolvendo, o comportamento de vinculação é normalmente dirigido para figuras não parentais (Weiss, 1982). Deste modo, o contexto dos pares e a relação amorosa assumem um papel cada vez mais determinante, como fonte de segurança interna, observando-se uma forte associação entre as relações interpessoais do adolescente e o autoconceito, o ajustamento psicológico e a saúde física (Bachman et al., 1975; Coopersmith, 1967; Greenberg et al., 1984; Thomas et al., 1974). É na adolescência que as relações com os pares começam a assumir um determinado número de funções que se irão manter por todo o desenvolvimento: fontes de intimidade, suporte, comparação e influência social e, por último, vinculação (Ainsworth, 1989).

A procura de autonomia dos pais pode funcionar como uma pressão saudável para o adolescente começar a utilizar os pares como figuras de vinculação, proporcionando um contexto onde as necessidades de vinculação podem ser satisfeitas, enquanto que a autonomia em relação aos pais 
está a ser estabelecida (Steinberg, 1990). A adolescência apresenta-se, assim, como uma etapa de vida em que as relações de vinculação são gradualmente transferidas para os pares, transferência esta que envolve a transformação das relações com os pais de uma posição hierárquica para uma interacção recíproca (Allen \& Land, 1999). Nesta investigação, procura-se avaliar diferencialmente a contribuição de dois importantes contextos de vida do jovem (relação com os pais e relação com o par amoroso), para a elaboração ou re-elaboração de um sentido interno de segurança pessoal.

Segundo Matos e Costa (1996), vinculação e autonomia são dois processos dialecticamente complementares e interdependentes, onde o processo de crescimento psicológico em direcção à autonomia é optimizado no contexto de proximidade emocional e de segurança transmitidos pela família. Neste contexto, as relações com os pares, apesar de, na adolescência, preencherem mais significativamente as necessidades de vinculação do sujeito, não funcionam como substitutas das suas relações com os pais; a relação do adolescente com os pais sofre progressivas transformações, no sentido da complementaridade para a reciprocidade, continuando a ser fundamental para a manutenção do seu sentido de segurança interna. A Teoria da Vinculação defende que os adolescentes seguros têm maior possibilidade de lidar, de um modo mais construtivo, com os desafios que acompanham os diferentes estádios do desenvolvimento psicológico.

Adoptando como quadro conceptual a teoria de Bartholomew e Horowitz (1991) sobre os estilos de vinculação, é possível considerar quatro estilos de vinculação, definidos a partir da combinação de duas dimensões (dicotomizadas como positivas ou negativas): o modelo do self e o modelo do outro. Estes quatro padrões de vinculação (o seguro, o preocupado, o amedrontado e o desinvestido) são conceptualizados como estratégias prototípicas de regulação emocional e de comportamento interpessoal em situações de proximidade afectiva. No estilo de vinculação seguro, situam-se aqueles indivíduos que possuem uma imagem positiva de si próprios e do outro, que têm capacidade para desenvolver e manter relações de intimidade sem perder a sua autonomia ou identidade, que não se preocupam em estar sós ou por existirem outros que não os aceitam. Os indivíduos preocupados são muito dependentes das relações que estabelecem para a manutenção da sua auto-estima, hiperenvolvendo-se nelas e definindo-se a partir delas; o seu bem-estar depende da total aceitação do outro. O estilo desinvestido caracteriza-se por uma independência e autoconfiança compulsivas, por uma repressão da partilha de emoções e sentimentos, que se traduz numa forte desvalorização da importância das relações de proximidade. Por fim, os indivíduos amedrontados, possuindo uma imagem negativa de si próprios e dos outros, evitam qualquer tipo de relação de proximidade por medo de serem rejeitados ou abandonados pelo 
outro. São indivíduos extremamente inseguros, com baixa autoconfiança, que simultaneamente desejam e temem a intimidade, confrontando-se com motivações conflituosas e ambivalentes.

A aquisição da autonomia e individuação marca a adopção de um novo estilo de vida pessoal e autónomo. É nesta fase que se denota uma maior capacidade para "se estar sozinho" e para encarar esses momentos como positivos e construtivos. Para os indivíduos seguros, os momentos em solitude podem representar um tempo utilizado pelos jovens para a construção da sua identidade, isto é, para explorar e experimentar opções alternativas respeitantes à identidade futura e reflectir sobre quem realmente são (Larson, 1995). Uma relação de vinculação segura promove no adolescente a criação de uma imagem positiva de si próprio e do outro, que o capacita para o desenvolvimento e a manutenção de relações de intimidade, sem a perda da própria autonomia ou identidade. Estes adolescentes não se preocupam em estar sós ou por existirem outros que não os aceitem, pois possuem um modelo de si próprios como pessoas capazes de ser amadas e respeitadas pelos outros.

\section{A influência da vinculação na solidão: hipóteses de estudo}

Os adolescentes são particularmente vulneráveis à solidão, dada a crescente importância da amizade nesta etapa desenvolvimental e o aumento da necessidade de intimidade e de self-disclosure, que podem ser satisfeitas por relações próximas com pares do mesmo sexo ou do sexo oposto (Brennam, 1982). Rubenstein e Shaver (1982) defendem que um dos factores atribuídos ao sentimento de solidão é a inexistência de uma vinculação segura que, na adolescência, se associa também à ausência de uma relação de intimidade. Os adolescentes com uma relação de vinculação segura aos pais apresentam menor dificuldade em estabelecer e manter relações de proximidade e, consecutivamente, menor solidão (Kerns \& Stevens, 1995).

Os modelos internos das figuras de vinculação, do self e do meio em geral, ao assumirem um papel activo na organização e orientação do comportamento da criança, e dos sentimentos e do processamento da informação nas relações que esta forma com os pais e pares, podem contribuir directamente para a solidão. As representações cognitivas que as crianças têm dos seus relacionamentos e vinculação aos pais vão influenciar as representações e relações que estas vão formar da relação com os pares, segundo um processo de generalização das mesmas, que pode ser alterado através das novas experiências de vinculação. Assim, são os modelos de representação que definem a segurança ou insegurança da vinculação, in- 
timamente relacionados com o modelo de representação do self que o indivíduo constrói, que o tornam mais ou menos vulnerável à solidão.

Os modelos internos dinâmicos são o construto-chave para a compreensão da relação entre a vinculação e a solidão: um modelo interno dinâmico do self como não susceptível de suscitar amor ou de ser valorizado pelos outros pode contribuir para a criação de sentimentos de solidão; um modelo da figura de vinculação como não disponível para satisfazer as necessidades de protecção e afecto do jovem podem deixá-lo num vazio emocional tão grande que ele experiencia um estado de solidão quase constante; por fim, um modelo que caracteriza o outro como rejeitante pode contribuir para que o indivíduo acredite que os outros não irão responder às suas necessidades ou desejos.

Os estilos de vinculação parecem exercer uma influência directa na solidão, pois um estilo de vinculação inseguro contribui para a manutenção de relações interpessoais insatisfatórias e para o isolamento social que, por sua vez, contribuem para a solidão. Diversos estudos que utilizaram medidas unidimensionais de solidão encontraram uma relação positiva entre uma vinculação insegura e a solidão em adolescentes e jovens adultos (Angelic \& Grigoris, 2000; DiTommaso, 1997; Goossens, Marcoen, Hees, \& Woestijne, 1998; Kerns \& Stevens, 1996; Kobak \& Sceery, 1988; Larose \& Bernier, 2001; Larose, Guay, \& Boivin, 2002; Man \& Hamid, 1998; Moller, McCarthy, \& Fouladi, 2002; Rice, 1990). Para Hecht e Baum (1984), o maior determinante da solidão não é a quantidade de tempo passado só, mas sim a ameaça de separação e a qualidade da experiência de vinculação. Deste modo, o factor determinante na emergência dos sentimentos de solidão não é a ausência da presença dos outros, mas o sentimento subjectivo de desvinculação emocional e a discrepância entre a necessidade de pertença e a incapacidade em a satisfazer.

Do nosso conhecimento, apenas dois estudos realizados até hoje estudaram a relação entre a solidão e a vinculação, utilizando medidas multidimensionais de solidão (DiTommaso, 1997; Goossens et al., 1998). A primeira investigação examinou a relação entre os estilos de vinculação (avaliados a partir do Relationship Scale Questionnaire, Griffin \& Bartholomew, 1994) e a solidão social, romântica e familiar (avaliada a partir da escala SELSA, DiTommaso \& Spinner, 1993) em mulheres, esposas dos membros das Forças Canadianas. Os resultados indicaram que níveis mais elevados de vinculação segura-amedrontada ${ }^{3}$ estavam associados a níveis menores dos três tipos de solidão.

\footnotetext{
${ }^{3}$ As subescalas que avaliavam o estilo seguro e amedrontado foram agrupadas numa só devido à elevada correlação existente entre elas, sendo designadas como um grupo de elevada segurança de vinculação.
} 
Os estudos realizados por Goossens et al. (1998) revelaram que as representações dos adolescentes das suas relações com os seus pais, de acordo com a Teoria da Vinculação, estão relacionadas com o grau de solidão que estes experienciam nos seus vários relacionamentos e com a atitude que estes assumem relativamente ao tempo que passam sozinhos. Os resultados indicaram que os adolescentes classificados como seguros (segundo o modelo de DeWuffel, 1986) experienciam níveis menores de solidão na relação com os pais e os pares; os dependentes são os que experienciam maior aversão à solitude; e os ansiosos-ambivalentes e evitantes são os que evidenciam atitudes mais positivas relativamente ao estado de solitude. Os dependentes ocupam uma posição intermédia entre os indivíduos seguros e os dois grupos de inseguros, no que se refere à solidão na relação com os pares e na procura de solitude.

Dada a evidência empírica, considera-se fundamental avaliar os diferentes níveis de solidão percepcionados pelos jovens nos diferentes contextos de vida (relação com os pais e relação amorosa) e analisar as atitudes que estes assumem relativamente ao estado de solitude (procura versus afastamento).

Este estudo procura analisar a relação entre a vinculação aos pais e ao par amoroso e os sentimentos de solidão, procurando compreender quais as dimensões da vinculação que estão implicadas na emergência dos sentimentos de solidão em cada contexto relacional e na atitude desenvolvida face ao tempo "passado só". Baseados no facto de que as diferentes relações que o indivíduo constrói e mantém contribuem de modo diferente e único para o seu sentido interno de segurança pessoal, distinguem-se três hipóteses de investigação: (1) a segurança da vinculação às figuras parentais e ao par amoroso irá exercer um efeito protector na percepção da solidão romântica; (2) a segurança da vinculação às figuras parentais e ao par amoroso irá exercer um efeito protector na percepção da solidão na relação com os pais; e (3) a segurança da vinculação aos pais e ao par amoroso irá exercer um efeito protector na emergência de atitudes negativas face à solitude (subescala Aversão da Solitude) e estará associada ao desenvolvimento de atitudes mais positivas face ao estar só (subescala Procura da Solitude).

\section{Metodologia}

\section{Amostra}

A amostra é constituída por 370 alunos de diferentes faculdades da Universidade do Porto e da Universidade Católica do Porto, com idades compreendidas entre os 18 e os 24 anos de idade $(\mathrm{M}=20,7$; $\mathrm{DP}=1,34)$. $69,8 \%$ da amostra é do sexo feminino e $30,2 \%$ é do sexo masculino. $\mathrm{Na}$ 
presente amostra, $58,3 \%$ dos participantes tem um namorado, contrariamente aos outros $41,7 \%$.

\section{Instrumentos de avaliação}

\section{Solidão}

A solidão foi avaliada através de uma adaptação de duas escalas de avaliação da solidão: a Louvain Loneliness and Aloneness Scale for Children and Adolescents (LACA; Marcoen et al.,1987) e a Social and Emotional Loneliness Scale for Adults (SELSA; Ditommaso \& Spinner, 1993). A LACA é constituída por 48 itens divididos em 4 subescalas, cada uma com 12 itens. Estas escalas avaliam respectivamente: a solidão nas relações com os pais (S-Pais), a solidão na relação com os pares (S-Pares), a aversão à solitude (Aversão) e a procura da solitude (Procura). Alguns itens destas escalas são: "Os meus pais dão-me apoio" (S-Pais), "Gostaria de estar melhor integrado na minha turma" (S-Pares), "Quando estou sozinho, sinto-me aborrecido" (Aversão) e "Eu quero estar sozinho" (Procura). As investigações que utilizaram este instrumento (Marcoen et al., 1987; Goossens, Marcoen, Van Hees \& Van de Woestijne, 1998; Goossens \& Marcoen, 1999a, 1999b) indicam que as 4 subescalas têm uma boa consistência interna $(0,76-0,88)$ e um grau de validade de construto moderado a elevado $(0,39-0,79)$. Esta escala foi utilizada na população portuguesa por Bastos, Figueira e Costa (2002).

A SELSA é constituída por 36 itens divididos por 3 subescalas, cada uma com 12 itens que avaliam, respectivamente, a solidão social (S-Social), a solidão emocional familiar (S-Familiar) e a solidão emocional romântica (S-Romântica). Este instrumento apresenta uma consistência interna excelente $(0,89$ to 0,93$)$ e boa validade concorrente, convergente e discriminante (DiTommaso \& Spinner, 1993; Fevens, Spinner \& Ditommaso, 1994). Desta escala apenas de se utilizou a subescala S-Romântica (exemplo de um item: "Eu dou por mim a desejar encontrar alguém com quem possa partilhar a minha vida") para avaliar a solidão romântica nesta investigação (S-Rom).

Os participantes responderam ao instrumento final numa escala de Likert de 5 pontos ( 1 - Discordo totalmente a 5 - Concordo totalmente). A análise em componentes principais através de rotação varimax permitiu determinar 5 factores, que reproduzem, quase na totalidade, a estrutura original da escala e explicam 49,5\% da variância total. A distribuição dos itens por cinco factores é consonante com as escalas originais, verificando-se uma excepção, atribuída a erros na tradução.

A consistência interna foi calculada através do índice alpha de Cronbach para cada um dos factores, verificando-se que cada subescala 
apresenta uma boa consistência interna (os alphas variam entre 0,75 e 0,95), sendo as correlações entre as 5 subescalas, no sentido e na extensão, semelhantes às encontradas pelos autores. Apesar da correlação negativa encontrada entre as subescalas Aversão e Procura, estas não devem ser consideradas diametralmente opostas, já que cada uma mede um aspecto diferente da experiência de solitude. Para este estudo, a subescala S-Pares não foi utilizada.

\section{Vinculação}

Vinculação Parental: O Questionário de Vinculação ao Pai e à Mãe (QVPM, Matos, Almeida \& Costa, 1998) permite avaliar as percepções que os adolescentes e os jovens adultos têm da sua vinculação ao pai e à mãe. O conteúdo dos itens foi desenvolvido teoricamente, de acordo com as contribuições de Bowlby $(1973,1977)$ e Ainsworth (1989) para o estudo da vinculação, e com modelo bidimensional de avaliação da vinculação adulta criado por Bartholomew (1991). Deste modo, o questionário mede 3 dimensões: Qualidade do laço emocional ("Eu e os meus pais temos uma relação de confiança"), Ansiedade de separação e dependência ("Não sou capaz de enfrentar situações difíceis sem os meus pais") e Inibição da exploração e individualismo ("Os meus pais impõem a maneira deles de ver as coisas"). A uma vinculação segura correspondem níveis elevados de qualidade de laço emocional, níveis reduzidos de inibição de exploração e dependência e de inibição da exploração e individualismo.

As qualidades psicométricas do instrumento suportam a sua validade, indicando uma elevada consistência interna (coeficiente alpha de Cronbach entre 0,74 e 0,88 e entre 0,68 e 0,89, para as escalas de vinculação à mãe e ao pai, respectivamente) e uma boa fidelidade teste-reteste $(0,54$ a 0,66$)$. A validade do auto-relato é ainda evidenciada pelas suas prováveis relações com outras medidas de avaliação da vinculação parental (Matos et al., 1999).

Os sujeitos responderam em escalas de Likert de seis pontos (1 "Discordo totalmente" e 6 - "Concordo totalmente"), classificando como sentem e pensam acerca de cada um dos seus progenitores. A análise em componentes principais, através de rotação varimax, permitiu determinar uma estrutura factorial composta por 30 itens, distribuídos por 3 factores, que explicam 56,1\% da variância total na versão Pai e 49\% na versão Mãe. Ambas as soluções factoriais fazem emergir três factores bipolares, teoricamente definidos pelas autoras (Matos et al., 1998).

A análise da consistência interna da escala QVPM, teoricamente definida por Matos (2001), revelou valores de alpha de Cronbach elevados (entre 0,85 e 0,94 ), semelhantes aos encontrados em estudos anteriores. 


\section{Vinculação Romântica}

O Questionário de Vinculação Amorosa (QVA, Matos, Barbosa \& Costa, 2001), construído para a população portuguesa, é constituído por 56 itens, organizados em quatro dimensões: Desconfiança no companheiro enquanto figura de vinculação ("O meu namorado desilude-me muitas vezes"), Evitamento ("Não costumo precisar do apoio do meu namorado"), Dependência ("Quando não podemos estar juntos, eu não sei o que fazer") e Ambivalência ("Tenho dúvidas se sou uma pessoa importante para ele"). O conteúdo dos itens foi desenvolvido teoricamente de acordo com as contribuições de Bowlby $(1973,1980)$ e Ainsworth (1989) para o estudo da vinculação, e como modelo bidimensional de avaliação da vinculação adulta criado por Bartholomew e Horowitz (1991). O instrumento pretende medir as percepções dos adolescentes e jovens adultos das suas relações de vinculação ao par amoroso, assim como ilustrar os quatro protótipos de vinculação definidos por Kim Bartholomew (1990). A uma vinculação segura correspondem níveis mais baixos de desconfiança, evitamento, dependência e ambivalência.

Os sujeitos responderam em escalas de Likert de seis pontos (1 "Discordo totalmente" e 6 - "Concordo totalmente"), classificando como sentem e pensam acerca de cada um dos seus progenitores. A análise em componentes principais através de rotação varimax originou uma estrutura factorial semelhante à original, composta por 51 itens, distribuídos por 4 factores, que explicam $45 \%$ da variância total. A escala revelou a adequação da estrutura prevista, sendo a distribuição dos itens por cada factor semelhante à encontrada pelas autoras do instrumento. A análise da consistência interna da escala QVA revelou valores de alpha de Cronbach elevados (entre 0,82 a 0,91 ), semelhantes aos encontrados em estudos anteriores.

\section{Resultados}

\section{Análises Correlacionais}

A análise das correlações (calculadas através do coeficiente de correlação de Pearson) existentes entre as variáveis demográficas e as subescalas de solidão demonstrou um efeito do género (codificado como $1=$ feminino e $0=$ masculino $)$ na solidão parental $(\mathrm{r}=-0,150, \mathrm{p}<0,01)$ e um efeito da variável existência de namorado na solidão romântica $(\mathrm{r}=-0,596, \mathrm{p}<0,01)$. 


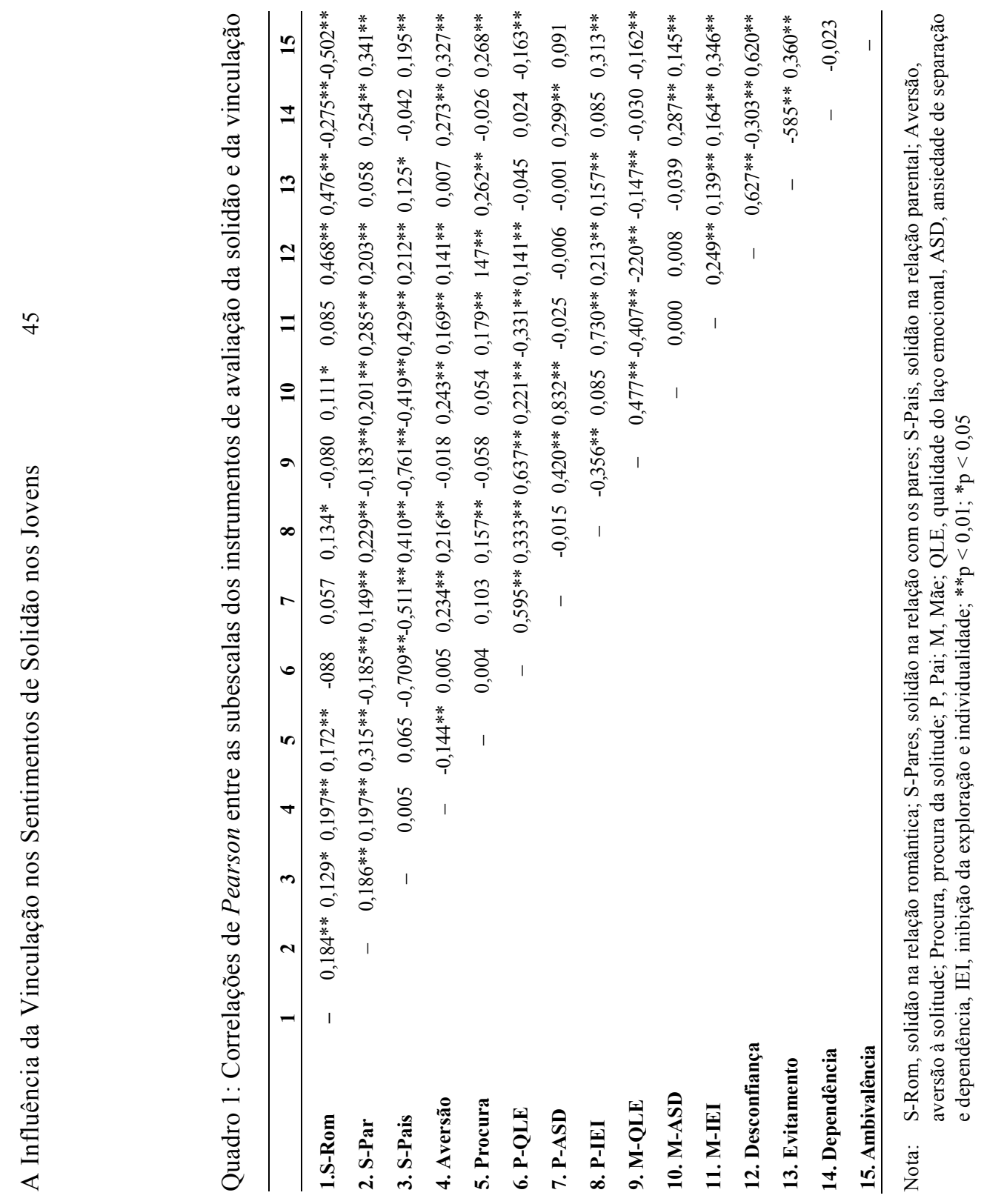


A análise das correlações existentes entre as subescalas de solidão e as dimensões da vinculação é apresentada no quadro 1. Como se pode verificar, diferentes dimensões de vinculação estão associadas a diferentes subescalas de solidão, sendo que as correlações mais elevadas ocorrem dentro dum mesmo domínio relacional (nomeadamente, relações parentais e relações românticas). A subescala Aversão surge correlacionada positivamente com a ansiedade de separação e a inibição da exploração ao pai e à mãe, e com a desconfiança, o evitamento e a ambivalência experienciados na relação com o par amoroso. A subescala Procura surge correlacionada positivamente com a inibição da exploração ao pai e à mãe, e com a desconfiança, a dependência e a ambivalência ao par amoroso.

De modo a melhor compreendermos a relação entre a vinculação e os sentimentos de solidão e as atitudes assumidas face à solitude, foram efectuadas quatro análises de regressão múltipla, segundo o método stepwise, uma para cada subescala de solidão (S-Romântica, S-Pais, Aversão e Procura). As várias dimensões de vinculação parental e romântica foram colocadas como variáveis independentes em todas as equações de regressão. A variável género foi também introduzida na análise de regressão efectuada para a solidão parental e a variável existência de namorado na análise de regressão efectuada para a solidão romântica.

Quadro 2: Variáveis preditoras de Solidão Romântica

\begin{tabular}{lccc}
\hline Variáveis independentes & $\mathbf{R}$ & $\mathbf{R}^{\mathbf{2}}$ & $\mathbf{R}^{\mathbf{2}}$ change \\
\hline Existência de relação de namoro & 0,844 & 0,712 & $0,712^{* *}$ \\
Ambivalência & 0,874 & 0,764 & $0,052^{* *}$ \\
Desconfiança & 0,877 & 0,769 & $0,005^{* *}$ \\
\hline
\end{tabular}

$* * \mathrm{p}<0,001$

A análise de regressão efectuada para a avaliação da primeira hipótese de investigação revela a existência de 3 variáveis que explicam significativamente $77 \%$ da variação nos sentimentos de solidão romântica: existência de namorado, ambivalência e desconfiança em relação ao par amoroso. A variável que melhor explica a solidão romântica é a existência de namorado, que explica $71 \%$ da variância total. 
Quadro 3: Variáveis preditoras de Solidão Parental

\begin{tabular}{lccc}
\hline Nome da Variável & $\mathbf{R}$ & $\mathbf{R}^{2}$ & $\mathbf{R}^{2}$ change \\
\hline M-QLE & 0,746 & 0,556 & $0,556^{* *}$ \\
P-QLE & 0,801 & 0,642 & $0,086^{* *}$ \\
P-IEI & 0,819 & 0,671 & $0,028^{* *}$ \\
M-ASD & 0,834 & 0,695 & $0,024^{* *}$ \\
\hline
\end{tabular}

$* * \mathrm{p}<0,001$

Relativamente à avaliação da vinculação na solidão parental (hipótese 2), observa-se que $70 \%$ da variação nos níveis de solidão é explicada por quatro variáveis, na seguinte ordem decrescente de importância: a qualidade do laço emocional à mãe, a qualidade do laço emocional ao pai, a inibição da exploração e individualidade pelo pai e a ansiedade de separação e dependência da mãe. A variável que explica a maior percentagem de variância (55.6\%) é a qualidade do laço emocional à mãe (ver Quadro 3).

Por último, as análises de regressão efectuadas para avaliar a terceira hipótese de investigação (apresentadas nos quadros 4 e 5), revelam que apenas uma percentagem pequena da variância nas atitudes face à solitude é explicada pelas dimensões da vinculação: $20,4 \%$ da variação nos resultados da Aversão à solitude é explicada pelas variáveis ambivalência e dependência ao par amoroso e ansiedade de separação e dependência ao pai; $13,3 \%$ da variação nos resultados da Procura de tempo para "estar só" é explicada pelas variáveis ambivalência, evitamento, desconfiança ao par amoroso e inibição da exploração e individualidade em relação à figura materna.

Quadro 4: Variáveis preditoras de Aversão à Solitude

\begin{tabular}{lccc}
\hline Nome da Variável & $\mathbf{R}$ & $\mathbf{R}^{2}$ & $\mathbf{R}^{2}$ change \\
\hline Ambivalência & 0,339 & 0,115 & $0,115^{* *}$ \\
Dependência & 0,428 & 0,183 & $0,068^{* *}$ \\
F-ASD & 0,452 & 0,204 & $0,021^{*}$ \\
\hline
\end{tabular}

${ }^{* *} \mathrm{p}<0.001 ; * \mathrm{p}<0.05$ 
Quadro 5: Variáveis preditoras de Procura de Solitude

\begin{tabular}{lccc}
\hline Nome da Variável & $\mathbf{R}$ & $\mathbf{R}^{2}$ & $\mathbf{R}^{2}$ change \\
\hline Ambivalência & 0,268 & 0,072 & $0,072^{* *}$ \\
Evitamento & 0,323 & 0,104 & $0,032^{* *}$ \\
M-IEI & 0,345 & 0,119 & $0,015^{*}$ \\
Desconfiança & 0,365 & 0,133 & $0,014^{*}$ \\
\hline
\end{tabular}

${ }^{* *} \mathrm{p}<0,001 ; *_{\mathrm{p}}<0,05$

\section{Discussão dos resultados e suas implicações para a intervenção}

Os resultados deste estudo permitem confirmar a primeira hipótese, ao demonstrarem o papel determinante da vinculação romântica e parental na experiência de sentimentos de solidão romântica. A existência de namorado provou ser a variável que exerce um maior efeito na percepção de solidão: os indivíduos que não possuem uma relação de namoro são os que percepcionam níveis mais elevados de solidão. Quando controlado o efeito desta variável, verifica-se que a ambivalência e a desconfiança na relação com o par amoroso são as dimensões responsáveis pela emergência de solidão. Ou seja, a solidão percepcionada numa relação amorosa está, em primeiro lugar, intimamente associada a sentimentos de insegurança e dúvida relativos ao papel que o sujeito representa enquanto companheiro amoroso e às suas próprias emoções face ao par; e, em segundo lugar, à sensação de possuir uma relação amorosa que não é suficientemente sensível e capaz de responder às necessidades de conforto e apoio do jovem, funcionando como uma base segura para a exploração. Sujeitos com valores mais elevados nestas dimensões da vinculação (por exemplo, indivíduos amedrontados ou desinvestidos) tenderão a isolar-se mais e a evitar o envolvimento genuíno nas relações de intimidade, por medo de serem rejeitados ou abandonados pelo outro. Por último, verifica-se que a qualidade da relação com o pai, percebida como fundamental e única ao desenvolvimento do sujeito, a quem este recorrerá em situações de dificuldade, tem também um peso, ainda que diminuto, na protecção da experiência de solidão na relação romântica.

A segunda hipótese de investigação foi parcialmente confirmada, verificando-se que a solidão avaliada na relação familiar é apenas explicada por dimensões associadas à vinculação parental. A qualidade da relação 
com a figura materna é a variável principal na protecção do adolescente contra a emergência da solidão, aparecendo, em segundo lugar, a qualidade da relação com o pai. A percepção de restrições impostas pelo pai à expressão de individualidade própria - seja pelas dificuldades sentidas na manifestação de valores ou opiniões, seja pela interferência não desejada em questões que o sujeito considera pessoais, ou ainda pela ausência de apoio a iniciativas de exploração - torna o indivíduo mais susceptível de experienciar solidão na relação com os pais. Por último, a experiência de ansiedade e medo de separação da figura materna, reveladora de uma relação de dependência, funciona como um factor protector da vivência de solidão. Consequentemente, os indivíduos com níveis elevados de ansiedade de separação e dependência e de qualidade de laço emocional, e com valores baixos de inibição da exploração e individualidade, deverão ser aqueles que experienciam níveis menores de solidão. Este padrão de resultados, nas três dimensões, parece evidenciar o protótipo preocupado, segundo a tipologia de Kim Bartholomew, tal como definido por Matos (2001). Estes indivíduos, ao evidenciarem uma grande dependência aos pais, ao procurarem a sua atenção e aprovação, limitando as suas acções de exploração autónoma, estarão sujeitos a níveis menores de solidão. No pólo oposto, encontramos os sujeitos com níveis elevados de inibição da exploração e níveis reduzidos de qualidade de laço emocional e ansiedade de separação e dependência, que serão os mais susceptíveis de sofrer de solidão na relação com os pais. Este padrão de resultados poderá evidenciar o protótipo desinvestido, caracterizado por uma independência e autoconfiança compulsiva, por uma repressão da partilha de emoções e sentimentos, que se traduz numa forte desvalorização da importância das relações de proximidade e, consequentemente, em maior solidão.

A qualidade da relação construída com o par amoroso, apesar de importante para a manutenção de um sentido interno de segurança pessoal do sujeito, não contribuiu para a solidão sentida na relação com os seus pais. Este factor poderá estar associado à qualidade da relação amorosa formada e ao facto de, na população portuguesa, se assistir a um fim cada vez mais tardio do processo de individuação e autonomia do adolescente - que o prepara para investir genuinamente na construção de relações com os pares e com o par amoroso -, variáveis cujo efeito deverá ser controlado num próximo estudo.

As análises efectuadas para explicar as atitudes que o indivíduo assume face à experiência de solitude mostram que uma parte significante da variação nos dados deve ser atribuída a factores não considerados neste estudo. Embora algumas dimensões da vinculação romântica e da vinculação parental estejam associadas ao modo como o indivíduo percepciona o 
tempo passado só, outras variáveis devem ser estudadas para melhor compreendermos o significado da solitude para o jovem.

No caso da subescala Aversão, verifica-se que uma relação de dependência (quer na relação com o companheiro amoroso, quer na relação com o pai) se associa a percepções mais negativas relativamente à solitude, já que o indivíduo necessita da proximidade do outro significativo para se definir e acreditar que é amado e valorizado pela figura de vinculação. Sentimentos de ambivalência na relação amorosa, aliados a uma atitude de dependência, poderão tornar o sujeito ainda mais incapaz de estar só, procurando o companheiro para tentar resolver os seus sentimentos de incerteza e dúvida face à relação amorosa que vivencia.

Por outro lado, na subescala Procura, os resultados demontraram que sentimentos de ambivalência, desconfiança e evitamento percepcionados na relação amorosa poderão promover uma atitude mais positiva face à solitude, necessária ao questionamento do papel do sujeito enquanto companheiro amoroso. Costa (1996) salienta que "A capacidade de estar só, sem se sentir só, é a pré-condição para saber amar e para a intimidade" (p. 10). Erickson (1980) reforça esta questão, ao afirmar que o investimento profundo numa relação de intimidade exige o autoconhecimento e a definição da identidade.

A existência de inibição da exploração e individualidade pela mãe parece também levar o indivíduo a procurar mais activamente momentos de solitude. Esses momentos permitir-lhe-ão explorar os seus gostos, os seus valores, o seu papel e função no mundo, necessários à aquisição de um sentido de unidade, continuidade e mutualidade, essenciais à definição da sua identidade (Costa, 1990). Goossens e Marcoen (1999) e Larson (1999) alertam para a importância da realização do processo de individuação e aquisição da autonomia para uma maior capacidade do indivíduo em "estar só" e em encarar esses momentos como positivos e construtivos.

Os resultados observados nesta investigação permitem-nos tecer algumas considerações para a intervenção clínica, alertando para a existência de um efeito protector da segurança da vinculação na emergência dos sentimentos de solidão. Nesta perspectiva, o trabalho terapêutico com adolescentes e jovens deve debruçar-se sobre as várias dimensões dos relacionamentos que cada um constrói, explorando os pressupostos subjacentes a cada um deles e o modo como as relações preestabelecidas influenciam a formação e o estabelecimento de novas relações significativas. É a qualidade da relação formada e a segurança que esta proporciona ao indivíduo que o vai proteger da experiência de sentimentos de solidão.

A necessidade de intervir na diminuição dos sentimentos de solidão foi já afirmada por diversos autores (Gordon, 1976; Tanner, 1973; Young, 1982). Fromm-Reichmann (1959) foi um dos primeiros autores a alertar os 
clínicos para uma conceptualização da solidão como uma problemática independente e clinicamente debilitante. McWhriter (1990) alertou para a importância de se considerar o tipo de solidão experienciada pelos indivíduos na investigação da eficácia das estratégias terapêuticas no tratamento da solidão. Um cliente que surge em consulta apresentando queixas de forte solidão a nível íntimo ou amoroso, pode ser muito diferente dum indivíduo que se sente só porque não consegue estabelecer uma relação segura com os seus pais, agora que atingiu a idade adulta. Os dados agora apresentados vêm reforçar esta questão, uma vez que, tal como se pode ver pela análise das correlações entre as cinco subescalas do instrumento de avaliação da solidão, estas representam factores distintos da experiência de solidão.

Ao longo dos anos, três tipos de abordagens têm sido utilizadas na intervenção nesta problemática: a terapia cognitivo-comportamental, o treino de skills sociais e o desenvolvimento de redes sociais de apoio (Rook \& Peplau, 1982).

Tendo em consideração a associação entre a solidão e as dimensões da vinculação aqui apresentadas, as intervenções cognitivo-comportamentais podem ser eficazes, ao modificar crenças disfuncionais e comportamentos autodebilitantes associados à solidão (Beck, Rush, Shaw, \& Emery, 1979, Young, 1982), mas não permitem ao sujeito a reconstrução de novos significados sobre si próprio e a sua história desenvolvimental, necessária à promoção de uma visão positiva de si próprio e do outro, característica dos indivíduos seguros. Já o treino de skills sociais, através da utilização de estratégias mais comportamentais centradas na resolução de problemas, pode encorajar o sujeito solitário a reflectir sobre as circunstâncias e factores que fizeram emergir os seus sentimentos de solidão. Ao incidirem sobre o modo como o sujeito inicia, mantém e termina os seus relacionamentos, estas terapias podem levá-lo a questionar-se sobre o modo como se posiciona em cada relacionamento, promovendo a diminuição de sentimentos de marginalidade, abandono, culpa e dúvida quanto às razões que levaram à dificuldade em estabelecer relacionamentos, ao seu término, à separação ou perda do outro significativo (Rook, 1984). O desenvolvimento de redes sociais de apoio pode também ser uma estratégia útil na diminuição dos sentimentos de solidão, ao proporcionar ao sujeito novas oportunidades e contextos de interacção social, onde ele se pode explorar, experimentar novos comportamentos, atitudes e reacções, aumentando o seu autoconhecimento, solidificando a sua auto-estima e reconstruindo activamente a sua identidade.

A utilização de todas estas estratégias deve, no entanto, ser implementada no contexto de consulta, onde a segurança proporcionada pela relação psicoterapêutica permite que o indivíduo se explore e confronte, 
sem o medo de perder a sua identidade, de ser julgado ou mal-interpretado. É apenas neste contexto que, muitas vezes, o adolescente encontra um espaço de reflexão pessoal, onde a certeza de que é aceite e apoiado the permite catalisar as suas energias para o seu autoconhecimento, o fortalecimento da sua identidade e autonomia, necessária ao estabelecimento de relações de vinculação seguras. Na exploração das relações de intimidade, deve ser explorado o modo como o sujeito se vê a si próprio enquanto suscetível de suscitar amor e protecção do outro e enquanto capaz de funcionar para ele como figura responsiva e sensível às suas necessidades.

$\mathrm{Na}$ consulta com o adolescente, também pode ser questionada a relação que este estabeleceu com as figuras parentais, promovendo-se a resolução adaptativa do seu processo de individuação. Os resultados deste estudo mostram que as dimensões mais importantes a trabalhar estão relacionadas com a importância da figura parental, enquanto fundamental e única no desenvolvimento do sujeito. Uma relação segura com os pais permitirá ao adolescente partir com confiança para o descobrimento do mundo que o rodeia, experimentando e construindo relações com outros significativos, sem medo de perder o afecto e amor dos pais.

A consulta deve ainda integrar os pais na intervenção, alertando-os para a importância de funcionarem como base segura ao processo de exploração dos filhos. A importância de serem capazes de "deixar partir", promovendo nos filhos a certeza que continuam emocional e psicologicamente junto deles, é necessária ao desenvolvimento adaptativo do processo de autonomia. É determinante alertar os pais para a necessidade que os adolescentes têm de "estarem sós" e de ver o seu espaço individual respeitado, explicando-lhes que este tempo lhes permitir reflectir e recontruir as suas significações pessoais. Neste processo, o papel do psicólogo passa por ajudar os pais a aceitar o crescimento físico e psicológico dos seus filhos, aprendendo a lidar com a perda daí resultante e promovendo condições que permitam reconstruir a relação com os filhos, no sentido de maior reciprocidade.

Concluindo, o modo como o jovem constrói as suas relações com os pais e outros significativos deve ser objecto de intervenção na psicoterapia com os indivíduos solitários, promovendo a construção e reconstrução de um sentido interno de segurança pessoal, necessário ao estabelecimento de relações securizantes e satisfatórias para o indivíduo. 


\section{Referências}

Ainsworth, M. (1989). Attachments beyond infancy. American Psychologist, 44, 709-716.

Allen, J. P., \& Land, D. (1999). Attachment in adolescence. In J. Cassidy \& P. R. Shaver (Eds.), Handbook of attachment: Theory, research and clinical applications (pp. 336-354). Nova Iorque: The Guilford Press.

Angelic, L., \& Grigoris, K. (2000). The relationship of parental attachment and psychological separation to the psychological functioning of young adults. Journal of Social Psychology, 140 (4), 451-466.

Asher, S. R., Hymel, S., \& Renshaw, P. (1984). Loneliness in children. Child Development, 55, 1456-1464.

Bartholomew, K. (1990). Avoidance of intimacy: An attachment perspective. Journal of Social and Personal Relationships, 7, 147-178.

Bartholomew, K., \& Horowitz, L. M. (1991). Attachment styles among young adults: A test of four-category model. Journal of Personality and Social Psychology, 61, 226-244.

Bastos, M. T., Figueira, F. O., \& Costa. M. E. (2001/2002). Avaliação da solidão nos jovens universitários portugueses. Cadernos de Consulta Psicológica, 17/18, 69-82.

Baumeister, R. S., \& Leary, M. R. (1995). The need to belong: Desire for interpersonal attachments as fundamental woman motivation. Psychology Bulletin, 3, 497-529.

Beck, A. T., Rush, A. J., Shaw, B. F., \& Emery, G. (1979). Cognitive theory of depression. Nova Iorque: Guilford Press.

Bérubé, I., \& Joshi, P. (1998). La Solitude et le concept de soi chez les jeunes et les stratégies adaptatives utilisées. Les Cahiers Internationaux de Psychologie Sociale, 37, 63-75.

Belcher, M. (1973). The measure of loneliness: A validation study of Belcher Extended Loneliness Scale (BELS). Dissertação de Doutoramento não publicada, EUA: Illinois Institute of Technology.

Bowlby, J. (1973). Attachment and loss: Separation, anxiety and anger. Nova Iorque: Basis Books.

Bowlby, J. (1980). Attachment and Loss: Loss, sadness and depression. Nova Iorque: Basis Books.

Bowlby, J. (1988). A secure base. Nova Iorque: Basis Books.

Brage, D., Meredith, W., \& Wooward, J. (1993). Correlates of Loneliness among Midwestern Adolescents. Adolescence, 28 (111), 685-693.

Brennan, T. (1982). Loneliness at adolescence. In L. A. Peplau \& D. Perlman (Eds.), Loneliness: A sourcebook of current theory, research and therapy (pp. 269-290). Nova Iorque: Wiley-Interscience. 
Buchholz, E., \& Catton, R. (1999). Adolescents' perceptions of aloneness and loneliness. Adolescence, 34 (133), 203-213.

Collins, N. S., \& Read, S. J. (1994). Cognitive Representations of attachment: The structure and function of working models. Advances in Personal relationships, 5, 53-90.

Costa, M. E. (1990). Desenvolvimento da identidade. In B. P. Campos (Ed.), Psicologia do desenvolvimento e educação de jovens (pp. 251-285). Lisboa: Universidade Aberta.

Costa, M. E. (1991). Contextos sociais de vida e desenvolvimento da identidade. Porto: Instituto Nacional de Investigação Científica / Centro de Psicologia da Universidade do Porto.

Costa, M. E. (1996). A intimidade à procura de um psicoterapeuta. Cadernos de Consulta Psicológica, 12, 5-12.

De Wuffel, F. J. (1986). Attachments beyond childhood: Individual and development differences in parent-adolescent attachment relacionships. Dissertação de Doutoramento não publicada. Holanda: Catholic University of Nijmegen.

DiTommaso, E. (1997). Assessing an attachment model of loneliness: The relationship between attachment style, chronic loneliness and coping. Dissertação de Doutoramento não publicada. EUA: The University of New Brunswick.

DiTommaso, E., \& Spinner, B. (1993). The development and initial validation of a measure of social and emotional loneliness (SELSA). Personality and Individual Differences, 14 (1), 127-134.

Erickson, E. (1980). Identity and the life cycle. Nova Iorque: Norton.

Fevens, A., Spinner, B., \& DiTommaso, E. (1994). Adult attachment style and mental health. Comunicação apresentada na Annual Convention of the Canadian Psychological Association, Penticton, Canada.

Fromm-Reichman, F. (1959). On loneliness. Psychiatry, 2, 1-15.

Gerstein, L. H., \& Tesser, A. (1987). Antecedents and responses associated with loneliness. Journal of Social and Personal Relationships, 4, 329-363.

Griffin, D., \& Bartholomew, K. (1994). The metaphysics of measurement: the case of adult attachment. In. K. Bartholomew and D. Perlman (Eds.), Advances in personal relationships: Attachment processes in adulthood (vol. 5, pp. 17-52). London: Jessica Kingsley.

Goossens, L., \& Marcoen, A. (1999). Relationship during adolescence: Constructive vs. negative themes and relational dissatisfaction. Journal of Adolescence, 22, 65-79.

Goossens, L., Marcoen, A., Hees, S. V., \& Woestijne, O. (1998). Attachment style and loneliness in adolescence. European Journal of Psychology of Education, $12,529-542$.

Greenberg, M. T., Siegal, J., \& Leitch, C. (1984). The nature and importance of attachment relationships to parents and peers during adolescence. Journal of Youth and Adolescence, 12 (5), 373-386.

Hazan, C., \& Shaver, P. R. (1987). Romantic love conceptualised as an attachment process. Journal of Personality and Social Psychology, 52, 511-524. 
Kerns, K. A., \& Stevens, A. C. (1996). Parent-child attachment in late adolescence: Links to social relations and personality. Journal of Young and Adolescence, 25,3 .

Kobak, R. R., \& Sceery, A. (1988). Attachment in late adolescence: Working models, affect regulation and representations of self and others. Child Development, 59, 135-146.

Larose, S., \& Bernier, A. (2001). Social support processes: Mediators of attachment state of mind and adjustment in late adolescence. Attachment \& Human Development, 3, 96-120.

Larose, S., Guay, F., \& Boivin, M. (2002). Attachment, social support, and loneliness in young adulthood: A test of two models. Society for Personality and Social Psychology, 28, 5, 684-693.

Larson, R. W. (1990). The solitary side of life: An examination of time people spend alone from childhood to old age. Developmental Review, 10, 155-183.

Larson, R. W. (1995). Secrets in the bedroom: Adolescents' private use of media. Journal of Youth and Adolescence, 24, 535-550.

Larson, R. W. (1999). The uses of loneliness. In K. J. Rotenberg \& S. Hymel (Eds.), Loneliness in childhood and adolescence (pp. 244-262). Nova Iorque: Cambridge University Press.

Main, K., Kaplan, N., \& Cassidy, J. (1985). Security in infancy, childhood and adulthood: A move to the level of representation. In I. Bretherton \& E. Waters (Eds.), Growing points in attachment theory and research. Monographs of the Society for Research in Child Development (vol. 50, 1-2, Serial Nr. 209, pp. 66-104).

Man, K., \& Hamid, N. (1998). The relationship between attachment prototypes, self-esteem, loneliness and causal attributions in Chinese trainee teachers. Personal Individual Differences, 24 (3), 357-371.

Marcoen, A., Goossens, L., \& Caes, P. (1987). Loneliness in pre-through late adolescence: Exploring the contributions of a multidimensional approach. Journal of Youth and Adolescence, 16 (6), 561-577.

Marcoen, A., \& Goossens, L. (1993). Loneliness, attitude towards aloneness, and solitude: Age differences and developmental significance during adolescence. In S. Jackson \& H. Rodriguez-Tomé (Eds.), Adolescence and its social worlds (pp. 197-227). Hove, Reino Unido: Erlbaum.

Matos, P. M. (2002). (Des)continuidades na vinculação aos pais e ao par amoroso nos adolescentes. Dissertação de Doutoramento não publicada. Porto: Universidade do Porto.

Matos, P. M., \& Costa, M. E. (1996). Vinculação e processos desenvolvimentais nos jovens e adultos. Cadernos de Consulta Psicológica, 12, 45-54.

Matos, P. M., Barbosa, S., \& Costa, M. E. (2001). Avaliação da vinculação amorosa em adolescentes e jovens adultos: Construção de um instrumento e estudos de validação. Revista Oficial de la Asociacón Iberoamericana de Diagnóstico y Evaluación Psicologica, II (1), 93-109.

Matos, P. M., Almeida, H., \& Costa, M. E. (1998). O questionário de vinculação ao pai e à mãe: desenvolvimento e estudos de validação. Poster apresentado na $6^{\mathrm{a}}$ 
Conferência Bienal da European Association for Research on Adolescence, Budapest, Hungria.

McWrither, B. T. (1990). Loneliness: A review of current literature, with implications for counselling and research. Journal of Counselling and Development, 68, 417-422.

Moller, N. P., McCarthy, C. J., \& Fouladi, R. T. (2002). Earned attachment security: Its relationship to coping resources and stress symptoms among college students following relationship break up. Journal of College Student Development, 43 (2), 213-230.

Perlman, D., \& Landolt, M. (1999). Examination of loneliness in children-adolescents and in adults: Two solitudes or a unified enterprise?. In K. J. Rotenberg \& S. Hymel (Eds.), Loneliness in childhood and adolescence (pp. 325-347). Nova Iorque: Cambridge University Press.

Perlman, D., \& Peplau, L. A. (1981). Toward a social psychology of loneliness. In R. Gilmour \& S. Duck (Eds.), Personal relationships: Personal relationships in disorder. (pp. 244-262) London: Academic Press.

Peplau, L. A., \& Perlman, D. (1982). Perspectives on loneliness. In L. A Peplau \& D. Perlman (Eds.), Loneliness: A sourcebook of current theory, research and therapy (pp. 1-18). Nova Iorque: Wiley-Interscience.

Rice, K. G. (1990). Attachment in adolescence: A narrative and meta-analytic review. Journal of Youth and Adolescence, 19, 511-538.

Rubenstein, M. C., \& Shaver, P. (1982). The experience of loneliness. In L. A. Peplau \& D. Perlman (Eds.), Loneliness: A sourcebook of current theory, research and therapy (pp. 206-223). Nova Iorque: Wiley-Interscience.

Russell, D., Peplau, L. A., \& Cutrona, C. E. (1980). The revised UCLA loneliness scale: Concurrent and discriminant validity evidence. Journal of Personality and Social Psychology, 46, 1313-1321.

Russell, D., Peplau, L. A., \& Ferguson, C. E. (1978). Developing a measure of loneliness. Journal of Personality Assessment, 42, 290-293.

Schmidt, N. (1976). The construction of a scale for the measurement of loneliness. Tese de Mestrado não publicada. Toronto: York University.

Steinberg, L. (1990). Autonomy, conflict, and harmony in the family relationship. In S. Feldman \& G. Elliot (Eds.), At the threshold: The developing adolescent (pp. 255-276). Cambridge, MA: Harvard University Press.

Sullivan, H. S. (1953). The interpersonal theory of psychiatry. Nova Iorque: Norton.

Weiss, R. S. (1973). Loneliness: The experience of emotional and social isolation. Cambridge, MA: MIT Press.

Weiss, R. S. (1982). Issues on the study of loneliness. In L. A. Peplau \& D. Perlman (Eds.), Loneliness: A sourcebook of current theory, research and therapy (pp. 71-80). Nova Iorque: Wiley-Interscience.

Winnicott, D. W. (1957). The capacity to be alone. International Journal of Psychoanalysis, 39, 416-420.

Young, J. E. (1982). Loneliness, depression and cognitive therapy: Theory and application. In L. A. Peplau \& D. Perlman (Eds.), Loneliness: A sourcebook of current theory, research and therapy (pp. 379-406) Nova Iorque: Wiley. 